\title{
Children with Special Health Care Needs: A Factual Perspective
}

\author{
Sapna Agrawal ${ }^{1}$ \\ ${ }^{I}$ Consultant Pediatric Dentist, Dental Department, Narayani Hospital Birgunj, Nepal.
}

\begin{abstract}
Children with special health care needs are present throughout the world, in all nations and all races. As oral health is an integral part of general health, these individuals are at an increased risk of acquiring oral diseases lifelong. Also, oral diseases can have a direct and devastating impact on their quality of life making daily activities more difficult if not treated on time. Managing and treating these differently ables requires increased awareness as well as attention along with specialized training. Establishing an early dental home along with the medical home would help improve their overall quality of life. A planned treatment protocol including proper referral system to the trained professionals would surely help in decreasing the load of oral diseases.
\end{abstract}

Key words: Management, pediatric dentistry, special health care needs (SHCN).

\section{INTRODUCTION}

The American Academy of Pediatric Dentistry (AAPD) recognizes that providing both primary and comprehensive preventive and therapeutic oral health care to individuals with special health care needs ( $\mathrm{SHCN})$ is an integral part of the specialty of pediatric dentistry. Special needs may include any physical, developmental, mental, sensory, behavioral, cognitive, or emotional impairment or limiting condition that requires medical management, health care intervention, and/or use of specialized services or programs. It may be congenital, developmental, or acquired through disease, trauma, or environmental cause and may impose limitations in performing daily selfmaintenance activities or substantial limitations in a major life activity. Health care for these individuals requires a specialized knowledge acquired by additional training,as well as increased awareness, attention, adaptation, and accommodative measures beyond what are considered routine. ${ }^{1}$

Management of children with special health care needs (SHCN) requires an interdisciplinary and collaborative effort between dentists, nutritionists, physicians, pediatricians, anesthesiologists and many other health

\section{Correspondence}

Dr. Sapna Agrawal

Consultant Pediatric Dentist

Dental Department, Narayani Hospital, Birgunj, Nepal

Email: drsapnaagrawal123@gmail.com

\section{Citation}

Agrawal S. Children with Special Health Care Needs: A Factual Perspective. J Nepalese Assoc Pediatr Dent. 2020;1(1):44-5. care professionals so as to provide an optimal care. Failure to accommodate patients with SHCN in a dental office could be considered as a discrimination so practitioners must provide physical access to an office (e.g., wheelchair ramps, disabled-parking spaces); however, they can face many barriers to obtaining optimal oral health care. ${ }^{2}$

Individuals with SHCN may be at an increased risk for oral diseases throughout their lifetime with a direct and devastating impact on the health and quality of life. Medications containing sugars, special diets, and need to eat frequently along with restricted manual dexterity make them more prone to have untreated dental caries along with poor oral hygiene. Behavior management is often challenging which often requires either in-office sedation or general anesthesia. In addition, the parental anxiety concerning the problems associated with a child's SHCN frequently delays their dental care until significant oral disease has developed.,

Because of the unmet dental care needs, emphasis on a dental home and comprehensive, coordinated services should be established where either dentists with additional trainings or pediatric dentists provide an opportunity to implement the individualized preventive oral health care thus, reducing risk for oral diseases.

These children often require special consideration when receiving dental treatment due to physical, medical, developmental or cognitive conditions. People with physical disabilities may have problems getting onto the dental chair. People with learning disabilities may become 
over anxious at the thought of going to the dentist or may need extra reassurance. People suffering from severe medical problems may need extra precautions or care. ${ }^{1}$

\section{ISSUES AND CHALLENGES IN MANAGING} CHILDREN WITH SPECIAL HEALTH CARE NEEDS

According to The World Report on Disability given by WHO, there are over one billion people with disabilities in the world. Among them, 110-190 million experience very significant difficulties corresponding to about $15 \%$ of the world's population. There is growing prevalence of disability mainly due to population ageing and the global increase in chronic health conditions. In a particular country, patterns of disability are influenced by trends in health conditions and environmental and other factorssuch as road traffic crashes, natural disasters, conflict, diet and substance abuse. ${ }^{5}$

\section{SPECIAL NEEDS IN CONTEXT OF NEPAL}

The estimates of disability in Nepal are found to vary considerably from one study to another. According to the Population Census of 2011, the overall prevalence of disability in Nepal was 2\%; with male disability of $2.2 \%$ and female disability $1.7 \%$. Physical disability was the most common type of disability, which accounts for more than one third of total disabilities. ${ }^{6}$

There are various organizations and schools in Nepal who work for the upliftment of children with special health care needs. They focus in every possible aspect from providing equal rights to quality education, thus improving their overall quality of life. There are currently twelve dental schools all over Nepal where pediatric dentists are working and providing best dental care to these children from their areas of reach. Kantipur Dental College and Hospital has a well-established department for differently abled children in Kathmandu.
Pediatric dentists all over Nepal have conducted many workshops, trainings and seminars that focus on care and management of special needs children. The Nepalese Association of Pediatric Dentistry (NAPD) has signed a MoU with Special Olympics Nepa on 4th of April 2019 to help level the inequalities and create an inclusive society through advocacy and has taken a huge step ahead to empower the athletes of Special Olympics Nepa with good oral health.

\section{CONGLUSION}

Caring for a special needs patient takes compassion, understanding and time. Professionals with the knowledge, skills and positive attitude must know about the special methods or techniques for prevention and treatment of oral health along with special dental treatment plans, methods, and technique modifications for the successful delivery of oral health care for these differently abled children.

General awareness to the public and parents either through mass media or dental school programs and continuing dental education must be done to reach the dental care needs of these special needs children who are special in their own way in order to obtain a need-based treatment. As pediatric dentists are the ones who provide comprehensive oral health care to these children, lobbying for adequate referral system by other practitioners has to be done once the patient's needs are beyond his/her skills in order to ensure the overall health of the patient.

If a child cannot learn the way we teach, maybe we should teach the way they learn - Ignacio Estrada

\section{Conflict of Interest: None}

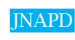

\section{REFERENCES}

1. American Academy of Pediatric Dentistry. Guideline on Management of Dental Patients with Special Health Care Needs. The Reference Manual of Pediatric Dentistry. 2012;34(5):160-5. [Full Text]

2. American Academy of Pediatric Dentistry. Management of dental patients with special health care needs. The Reference Manual of Pediatric Dentistry. Chicago, Ill.: American Academy of Pediatric Dentistry. 2020:275-80. [Full Text]

3. Avery DR and McDonald RE. Dentistry for the child and adolescent. 10 $0^{\text {th }}$ edition. 2016 Mosby. In Chapter 25, Dental Problems of children with Special Health Care Needs: 513-539. [Link]

4. Wright GZ and Kupietzky A. Behavior management in dentistry for children. $2^{\text {nd }}$ edition. 2014. John Wiley \& Sons. In Chapter 7, Children with Disabilities: 93-106. [Link

5. World report on Disability. 2011. WHO. Retrieved 2 October 2020. Available from: https://www.who.int/disabilities/world_report/2011/report.pdf. [Link]

6. Khanal SP. Persons with Disability and Their Characteristics. Population Monograph of Nepal. 2014;II(9):351-373. [Full Text] 